\title{
POTENCIAL MUTAGÉNICO Y GENOTÓXICO DE AGUAS RESIDUALES DE LA CURTIEMBRE TASAJERO EN LA CIUDAD DE CÚCUTA, NORTE DE SANTANDER, COLOMBIA
}

\section{MUTAGENIC AND GENOTOXIC POTENTIAL OF WASTE WATER FROM THE TANNERY TASAJERO, CUCUTA, NORTH SANTANDER, COLOMBIA}

\begin{abstract}
Alfonso Quijano Parra ${ }^{1}$, Carol Castillo T. ${ }^{2}$, Iván Meléndez Gélvez ${ }^{3}$
${ }^{1}$ Químico, Ph.D. Universidad de Pamplona. Profesor Asociado. Grupo de investigación en química. Laboratorio de control de calidad. Departamento de química y biología. Facultad de ciencias básicas. alfonsoquijanoparra@unipamplona.edu.co; ${ }^{2}$ Especialista en Bioquímica. Grupo de Investigación en biología molecular. Facultad de Ciencias Básicas. Universidad de Pamplona. carolcas@unipamplona.edu.co; ${ }^{3}$ Biologo, M.Sc. Universidad de Pamplona. Profesor asistente. Departamento de Química y Biología. Facultad de Ciencias Básicas. Grupo de Investigación en Biología Molecular -Biomogen; imgelvez@ unipamplona.edu.co; Ciudadela Universitaria, km 1, Vía Bucaramanga, Pamplona, Norte de Santander, Colombia; Correspondencia: alfonsoquijanoparra@unipamplona.edu.co
\end{abstract}

Rev. U.D.C.A Act. \& Div. Cient. 18(1): 13-20, Enero-Junio, 2015

\section{RESUMEN}

La curtición es un proceso industrial que requiere grandes volúmenes de agua para la transformación de un material putrescible en uno imputrescible, apto para su utilización, en productos manufacturados. Las aguas residuales generadas por dicha industria, generalmente, son vertidas a los cuerpos de agua sin tratamiento previo, generando así grandes cantidades de demandas bioquímicas de oxígeno y química de oxígeno, valores elevados de $\mathrm{pH}$, presencia de sustancias químicas tóxicas, que actúan como mutágenos, aumentando así el riesgo de desarrollar cáncer en la población expuesta. En la ciudad de Cúcuta, se encuentran pequeñas industrias de curtiembres, que no poseen las herramientas básicas, ni conocimientos sobre el adecuado tratamiento de los residuos, generando contaminación en el recurso hídrico, que luego puede llegar a ser usado por la población. En los procesos químicos de esta industria, se usan el cromo, el plomo, los sulfuros, los cromatos de zinc y el calcio, compuestos considerados, entre los carcinógenos humanos, más potentes. El estudio contempló el análisis y la determinación de la mutagenicidad y genotoxicidad en aguas residuales de la curtiembre Tasajero. Para la determinación de la genotoxicidad, se usó el ensayo cometa y para el análisis de la mutagenicidad, se aplicó el test de Ames, con las cepas de Salmonella typhimurium TA98 y TA100. Se encontró que las aguas de desecho de la curtiembre Tasajero indu- cen daño al ADN en linfocitos humanos; de igual manera, se halló que estas aguas también inducen un incremento en la mutagenicidad en la cepa TA98.

Palabras clave: Salmonella typhimurium TA98, TA100, metales pesados, test de Ames, ensayo cometa.

\section{SUMMARY}

Tanning is an industrial process that requires large volumes of water for the transformation of a putrescible material into a non-putrescible one, suitable to be used in manufactured products. The wastewater generated by this industry are generally discharged without previous treatment into water bodies, generating large amounts of biochemical demands of oxygen and chemical oxygen, high $\mathrm{pH}$ values, presence of toxic chemical substances that act as mutagens, increasing the risk of developing cancer in the exposed population. In the town of Cúcuta small tannery industries exist, which do not dispose the basic tools or knowledge regarding the proper waste treatment, therefore generating pollution of water, which afterwards can be used by the population. In the chemical process of this industry, chromium, lead, sulfides, zinc chromates and calcium, compounds considered among the most potent human carcinogens are used. The study included the analysis and determination of the mutagenicity and genotoxicity of wastewater from the tannery "Tasajero". 
For genotoxicity determination the comet assay was used; for the mutagenicity analysis, the Ames test with Salmonella typhimurium strains TA98 and TA100 was employed. It was found that the wastewater from the evaluated tannery induces DNA damage in human lymphocytes. Similarly, the results indicate that this water increased the mutagenicity in the TA98 strain.

Key words: Salmonella typhimurium TA 98, TA100, heavy metals, Ames test, comet assay.

\section{INTRODUCCIÓN}

La industria del curtido de cuero es conocida por ser uno de los principales sectores económicos de muchos países. Ha habido una creciente preocupación ambiental en relación con la liberación de varios contaminantes en aguas residuales de curtiembres (Lofrano et al. 2013). La Unión Europea es uno de los productores líderes de cuero; se estima que entre 400.000 y 900.000 toneladas de lodos se genera anualmente en la UE, a partir del procesamiento de cuero, la mayoría, de los cuales se depositan en vertederos (European Comission, 2001). Dhayalan et al. (2007) reportaron que, alrededor de 170.000 toneladas de residuos de piel curtida, se generan anualmente en la Unión Europea, por lo tanto, la eliminación de residuos generados en la producción de cuero es un problema que requiere medidas tecnológicamente avanzadas, tendientes a combatirlo (Thanikaivelan et al. 2005).

El curtido consiste en la conversión de las pieles de animales sin procesar en cuero, a través de una serie de procesos químicos, que requieren de grandes volúmenes de agua (Törnqvist et al. 2011). En el proceso de curtido, se emplean productos químicos, como cloruro de sodio, hidróxido de sodio, hipoclorito de sodio, agentes tenso activos, preparaciones enzimáticas, ácidos orgánicos tamponados, sulfúrico, clorhídrico, láctico, fórmico, bórico y mezclas, sales de amonio, siendo las sales de cromo las más utilizadas (Di laconi et al. 2002); bisulfito de sodio, peróxido de hidrógeno; solventes como kerosene y el clorobenceno; curtientes sintéticos, como los sintanos, anilinas, polímeros acrílicos o poliuretanos, lacas nitrocelulósicas o uretánicas.

Las aguas residuales generadas por las curtiembres contienen grandes concentraciones de cromo, en forma de dicromato de potasio $\mathrm{K}_{2} \mathrm{Cr}_{2} \mathrm{O}_{7}$ y sulfato de cromo $\mathrm{Cr}_{2}\left(\mathrm{SO}_{4}\right)_{3}$, que son altamente tóxicos (Álvarez et al. 2006); estos compuestos requieren, de alguna forma, tratamiento biológico, para permitir la eliminación segura al medio ambiente (Dotro et al. 2012).

$\mathrm{El} \mathrm{Cr}(\mathrm{VI})$ induce respuesta inmunológica inflamatoria y alteración de las vías de señalización de supervivencia celular. El $\mathrm{Cr}(\mathrm{VI})$ entra a la célula, a través de canales aniónicos no es- pecíficos y es metabólicamente reducido por agentes, como el ascorbato, glutatión y cisteína a $\mathrm{Cr}(\mathrm{V}), \mathrm{Cr}$ (IV) y $\mathrm{Cr}$ (III).

El Cr(III) es poco permeable a la membrana, por lo que es incapaz de atravesarla; este hecho, posibilita la unión con el ADN, generando daño genético, que conduce a la inestabilidad genómica. Estudios epidemiológicos realizados en el Reino Unido, en Japón y en Norte América, han mostrado que trabajadores expuestos al cromo, tienen un elevado riesgo de padecer enfermedades respiratorias, fibrosis, perforación del septo nasal, pólipos nasales y cáncer de pulmón (Plunkett, 1976; Witschi et al. 2008).

La exposición ambiental e industrial a los compuestos de cromo ha conducido al Instituto Nacional de Seguridad Ocupacional y Salud (NIOSH) a incluir el cromo como uno de los mayores causantes de cáncer de pulmón, por exposición ocupacional; a la Agencia de Protección Ambiental de EUA y la Agencia Internacional para la Investigación en Cáncer (IARC), a clasificarlo como un carcinógeno humano, resaltando al $\mathrm{Cr}(\mathrm{VI})$, como uno de los 33 compuestos potencialmente lesivos para la salud humana, en zonas urbanas (U.S. EPA, 1999; Agency for Toxic Substances and Disease Registry ATSDR, 2000; National Toxicology Program, 2005). Actualmente, es muy discutido el riesgo de ingestión del $\mathrm{Cr}(\mathrm{VI})$ en aguas de consumo. Estudios in vivo han mostrado que el $\mathrm{Cr}(\mathrm{VI})$ es un genotóxico marcadamente dependiente de la dosis, con la evidencia de un fuerte efecto umbral, debido a la desintoxicación extracelular por reducción a $\mathrm{Cr}(\mathrm{III})$, antes de la absorción por órganos y tejidos periféricos (De Flora, 2000).

Estudios en ratas y ratones muestran aberraciones cromosómicas en médula ósea, ruptura de cadena simple en ADN de hígado y cerebro, como también ruptura de cadena simple de ADN en leucocitos (Acharya et al. 2001; Sedman et al. 2006). Ciertas formas de $\mathrm{Cr}(\mathrm{VI})$ son conocidas como carcinógenos respiratorios, que inducen un amplio espectro de daños en el ADN (O'Brien et al. 2001; Salnikow \& Zhitkovich, 2008). Lesiones genéticas estructurales producidas por el $\mathrm{Cr}(\mathrm{VI})$ inducen aductos, rupturas, uniones proteína-ADN, oxidación de bases, sitios AP y entrecruzamientos entre cromátidas (O’Brien et al. 2003; Ceryak et al. 2004). La asociación del cromo con el enlace fosfodiester es una de las lesiones primarias que origina mutagenicidad en células de mamíferos (Salnikow \& Zhitkovich, 2008). El daño inducido por cromo conduce a la replicación disfuncional del ADN, causando sustitución y delección de bases, que son sustrato para los sistemas de reparación por escisión de bases (BER) y reparación por escisión de nucleótidos (NER), promoviendo así la inestabilidad genómica (O’Brien et al. 2009).

Hay varias líneas de evidencia que sugieren la importancia de la sustitución de bases en la mutagenicidad del cromo, sien- 
do la sustitución Guanina: Citosina el blanco primario, tanto in vivo como in vitro (Holmes et al. 2008). La reducción del $\mathrm{Cr}(\mathrm{VI})$ ha sido mostrada para producir especies reactivas, tales como radicales libres, anión superoxido y radicales hidroxil, posiblemente, a través de la reacciones del $\mathrm{Cr}(\mathrm{V})$ y $\mathrm{Cr}$ (IV) con el peróxido de hidrógeno, similares a la reacción de Fenton (Jennette, 1982; Sedman et al. 2006). Además, se ha encontrado que en presencia de vitamina A, en células V79, se incrementa el radical hidroxilo, las aberracciones cromosómicas y las mutaciones sobre el locus HGPRT (Sugiyama, 1991; Sugiyama et al. 1992).

El daño inducido por el $\mathrm{Cr}(\mathrm{VI})$ conduce a la replicación disfuncional del ADN, desregulación de sitios de control del ciclo celular, desregulación de sistemas de reparación, inestabilidad de microsatélites, respuesta inflamatoria y el daño de genes, clave en la regulación de redes responsables del balance, entre la vida y la muerte celular, que juegan un papel importante en la carcinogénesis del $\mathrm{Cr}(\mathrm{VI})$. Varias líneas de evidencia indican que la progresión neoplásica es el resultado de cambios epigenéticos y genéticos, que confieren a la célula ventajas de supervivencia que, en último término, conducen a la conversión de una célula normal a una célula maligna. La vía de absorción cutánea es la de más fácil acceso al organismo, frente a lo cual, cabe destacar el cuadro patológico de la dermatitis alérgica de contacto. En ésta, los compuestos de $\mathrm{Cr}(\mathrm{VI})$ penetran en la piel más rápidamente que los compuestos de Cr(III) (Chávez Porras, 2010).

En el área metropolitana de Cúcuta existen curtiembres que no tratan adecuadamente los residuos, generando contaminación ambiental. Estas industrias utilizan en el proceso de curtido del cuero cromatos de zinc y calcio, considerados como potentes carcinógenos en humanos (Wise et al. 2004; Shakir et al. 2012). La exposición intensa a estos compuestos ha producido una incidencia muy elevada de cáncer de pulmón (Castleman, 1995; Alberg \& Samet, 2003).

El objetivo de la presente investigación fue determinar la mutagenicidad y genotoxicidad en aguas residuales, de una curtiembre en la ciudad de Cúcuta, Norte de Santander.

\section{MATERIALES Y MÉTODOS}

La muestra de agua residual en la curtiembre Tasajero de la ciudad de Cúcuta, Norte de Santander, se recolectó en un botellón de polietileno, previamente lavado, en una cantidad aproximada de 20L; se obtuvo directamente de los vertimientos realizados después del proceso de desencalado, que consiste en eliminar los residuos químicos depiladores, como las sales de calcio o cal del cuero, utilizando cloruro de amonio o bisulfito de sodio y, de esta manera, evitar que en el proceso se formen sales insolubles y disminuir el exceso de sulfuro de hidrogeno, cuando se, reduzca el pH. En la curtiembre Tasajero en el proceso de desencalado, se utilizan sales de sulfato y cloruro de amonio así como bisulfito de sodio.

Concentración de la muestra de agua residual: Inicialmente, los $20 \mathrm{~L}$ de agua residual de la curtiembre se dejó reposar, durante seis horas, a una temperatura de $6^{\circ} \mathrm{C}$ y se pasó por un filtro de tela, para eliminar las impurezas gruesas. Para la concentración de la muestra, se pasaron 20L de agua residual, a una velocidad de $15 \mathrm{~mL} / \mathrm{min}$, por una columna que contenía $100 \mathrm{~g}$ de resina Amberlite XAD-2, de marca Supelco; después de pasar toda la muestra, la columna se eluyó con diclorometano. Posteriormente, la muestra se concentró en un rotaevaporador, a una temperatura de $60^{\circ} \mathrm{C}$, a $150 \mathrm{rpm}$, hasta llevarla a sequedad. Seguidamente, se diluyó el residuo en $20 \mathrm{~mL}$ de dimetilsulfoxido al $12 \%$, para conservarla.

Detección de la actividad mutagénica: El efecto mutagénico de los extractos de las aguas residuales de la curtiembre, se determinó por medio del test de Ames (Ames et al. 1973), usando el protocolo revisado por Maron \& Ames (1973). En esta prueba, el indicativo de la mutación es la reversión de his a his ${ }^{+}$; los revertantes, se conocen porque crecen en medio mínimo sin histidina. Se trabajó con dos cepas de Salmonella thypimurium (Tabla 1). En la cepa TA98, se produce la reversión a his ${ }^{+}$por perdida o ganancia en un par de bases en el gen mutado y, en la cepa TA100, se produce la reversión por sustitución de un par de bases. Con cada muestra se trataron $10^{7}$ bacterias, en ausencia de enzimas microsomales. Se utilizaron controles positivo y negativo; para el control positivo, se manejó 4-nitroquinolina oxido (4$\mathrm{NQO}$ ) y como control negativo, dimetilsulfoxido (DMSO), al $12 \%$. Se consideraron positivos los resultados que presentaron, de forma reproducible, un incremento significativo de la mutación con respecto al control negativo. Para verificar la reproducibilidad de los resultados, se hicieron tres experimentos independientes, cada uno por duplicado. De cada uno de los extractos, se analizaron dosis subtoxicas, las cuales, se determinaron haciendo curvas de mutagenicidad, con dosis pequeñas, medianas y altas. Las dosis subtoxicas que se utilizaron fueron 50,100 y $150 \mu \mathrm{g}$ del extracto total.

Detección del daño del ADN: Para detectar la ruptura del ADN, se utilizó el ensayo cometa. Se siguió la metodología propuesta por Singh et al. (1988) y modificada por Pandrangi et al. (1995). La ocurrencia de daño en el ADN, se basó en la longitud de la cola del cometa, inducida por la ruptura del ADN. Se consideró que la cola era producida por ruptura, si era mayor de $26 \mu \mathrm{m}$, que es la longitud producida por factores espontáneos. Para garantizar que el daño genotoxico observado es debido a los compuestos presentes en el extracto y no a eventos biológicos, como la apoptosis, se analizaron dosis subtóxicas de 50, 100 y150 $\mu \mathrm{g}$. 
Tabla 1. Características de la Salmonella typhimurium utilizada en este estudio.

\begin{tabular}{|c|c|c|}
\hline Cepa & Marcadores genéticos & Referencia \\
\hline TA-98 & $\begin{array}{l}\text { uvrB } \\
\text { hisD3052 } \\
\text { bio } \\
\text { rfa } \\
\text { R-factor } \\
\text { plasmido-pkM101 } \\
\text { Mutación por desplazamiento del marco de lectura en sitios } \\
\text { G-C }\end{array}$ & Ames et al. (1975) \\
\hline TA-100 & $\begin{array}{l}\text { uvrB } \\
\text { hisG46 } \\
\text { bio } \\
\text { rfa } \\
\text { R-factor } \\
\text { plasmid-pkM101 } \\
\text { Mutación por sustitución de bases en sitios G-C }\end{array}$ & Ames et al. (1975) \\
\hline
\end{tabular}

Extracción de linfocitos: Para los experimentos de genotoxicidad, se usaron linfocitos de sangre periférica, que se extrajeron de una persona joven y sana. Los linfocitos se separaron de la sangre total, usando un gradiente de ficollhipaque. Seguidamente, se determinó la viabilidad, la cual, siempre se mantuvo por encima del $95 \%$.

Tratamiento: Se tomaron $200 \mu \mathrm{L}$ de células, que se mezclaron con $50 \mu \mathrm{L}$ del tratamiento o control; posteriormente, se incubó durante $1 \mathrm{~h}$, a $37^{\circ} \mathrm{C}$; luego se tomó $75 \mu \mathrm{L}$ agarosa de bajo punto de fusión y se mezcló con $10 \mu \mathrm{L}$ de células tratadas, para luego ser vertidas en una lámina base de agarosa de punto de fusión normal; seguidamente, se llevó a incubar 6 min a $4^{\circ} \mathrm{C}$; a continuación, se retiró el cubre objeto y se adicionaron otros $75 \mu \mathrm{L}$ de agarosa e incubó $6 \mathrm{~min}$, a $4^{\circ} \mathrm{C}$.
Terminada la incubación, las láminas base se sumergieron en solución de lisis mínimo 1h; posteriormente, se lavaron las placas con PBS y se procedió a realizar la electroforesis por 30 minutos, sin conectar a la fuente de poder y 30 minutos, conectada a $25 \mathrm{v}$ y $300 \mathrm{~mA}$; luego, las placas se lavaron con solución neutralizante. Una vez terminado el proceso, se procedió a la coloración con bromuro de etidio y a la observación en el microscopio de fluorescencia Olympus U-RFKT50, con el objetivo de 25X; se analizaron un total de 200 células

\section{RESULTADOS Y DISCUSIÓN}

En la tabla 2 se muestra la razón de mutagenicidad inducida por los extractos de las aguas residuales de la curtiembre Tasajero.

Tabla 2. Razón de mutagenicidad calculada a partir de los datos de mutagenicidad de dos experimentos con la cepa TA98 y TA100, inducida por extractos de aguas residuales de la curtiembre Tasajero de la ciudad de Cúcuta.

\begin{tabular}{|c|c|c|}
\hline \multicolumn{3}{|c|}{ Razón de mutagenicidad } \\
\hline Dosis en $\boldsymbol{\mu g}$ & TA98 & TA100 \\
\hline 50 & 3,5 & 1,41 \\
\hline 100 & 3,8 & 1,52 \\
\hline 150 & 4,4 & 1,65 \\
\hline DMSO (12\%) & 1 & 1 \\
\hline
\end{tabular}

DMSO dimetilsulfoxido. La razón de mutagenìcidad (RM) es igual a los revertantes inducidos, por el tratamiento (RI) sobre los revertantes inducidos, por el control o revertantes espontaneos (RE). 
La razón de mutagenìcidad (RM) es igual a los revertantes inducidos por el tratamiento (RI) sobre los revertantes inducidos por el control o revertantes espontáneos (RE) así: $\mathrm{R} M=\mathrm{RI} / \mathrm{RE}$.

De acuerdo a las razones de mutagenicidad mostradas en la tabla 2, se considera que la muestra es positiva para la cepa TA98, ya que existe una relación de dosis de respuesta entre las concentraciones probadas y el número de revertantes inducidos; esto se puede determinar utilizando los criterios descritos en Vargas et al. (2001), donde la muestra se considera mutagénica cuando el número de colonias revertantes es $\geq 2$. Además, la significancia de la reversión en las cepas ensayadas fue determinada, a través de análisis de varianza a una vía, que muestran que la reversión incrementa significativamente $(\mathrm{p} \leq 0.05)$, a medida que aumenta la dosis La mutagenicidad observada se puede atribuir a la presencia de metales pesados, tales como $\mathrm{Cr}$ y $\mathrm{Hg}$, que son muy comunes en las aguas de residuo de las curtiembres, según lo plantea Tagliari et al. (2004). De acuerdo con los resultados mostrados en la tabla 2, en el caso de la cepa TA98, se observa que cada una de las dosis supera entre tres y cuatro veces el valor del control negativo, mientras que en la cepa
TA100, no se observa un incremento de la razón de mutagenicidad superior a dos, de acuerdo con Vargas et al. (2001), lo cual indica que los compuestos vertidos por esta industria inducen mutación por pérdida o ganancia de bases, más que por sustitución de bases.

En el ensayo COMETA, se obtuvo un promedio de tres experimentos individuales y, en cada uno de ellos, se analizaron 150 células, para así determinar, con base en la longitud en $\mu \mathrm{m}$, la migración de los fragmentos del ADN, como se puede ver en la tabla 3 . De acuerdo a los valores obtenidos en cada una de las dosis y comparados con el control negativo, se notó una tendencia creciente de daño genotóxico según el aumento de la concentración, conllevando a un incremento de las lesiones primarias sobre el ADN, lo cual, está directamente relacionado con el aumento en las alteraciones genéticas celulares. En la figura 1, se detallan fotografías de algunos núcleos, después de ser sometidos a diferentes concentraciones del extracto de aguas residuales de la curtiembre Tasajero de Cúcuta. Como se puede observar, a medida que se incrementa la dosis, el daño, visualizado como cola del cometa, es mayor.

Tabla 3. Daño del ADN en linfocitos de sangre periférica humana, inducida por el extracto orgánico del agua de la curtiembre Tasajero.

\begin{tabular}{|c|c|}
\hline Dosis $(\mu \mathrm{g})$ & X promedio $(\mu \mathrm{m})$ \\
\hline 50 & 40 \\
\hline 100 & 66 \\
\hline 150 & 73 \\
\hline PBS & 26 \\
\hline $\mathrm{H}_{2} \mathrm{O}_{2} 25 \mathrm{mM}$ & 83 \\
\hline
\end{tabular}

El daño se cuantificó como el promedio de la longitud de la cola en 150 células analizadas. Se realizaron tres ensayos cada uno por duplicado. Como control positivo, se usó peróxido de hidrógeno $25 \mathrm{mM}$, como control negativo DMSO al $1 \%$.

Figura 1. Imágenes del daño inducido en linfocitos humanos por muestras de agua residual de la curtiembre Tasajero de Cúcuta, Norte de Santander.
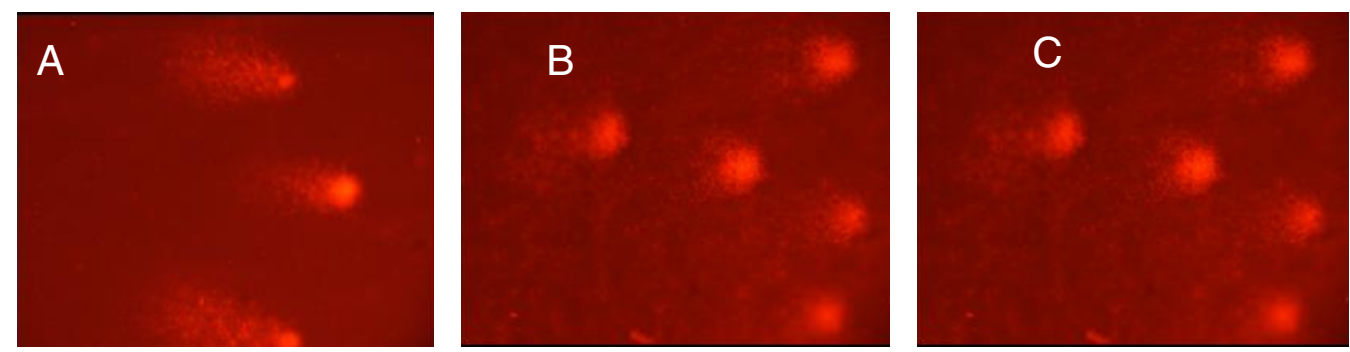

A: daño inducido por la fracción 3 dosis de $50 \mu \mathrm{g}$, B: daño inducido por la fracción 2 dosis de $50 \mu \mathrm{g}$,

C: daño inducido por la fracción 1 dosis de $50 \mu \mathrm{g}$. 
Se encontró, que cada una de las dosis ensayadas aporta sustancialmente daño al ADN en linfocitos humanos, expuestos a los compuestos presentes en las aguas residuales de curtiembres, probablemente, debido a la presencia de nitro-aromáticos, así como también de metales, como $\mathrm{Cr}$, $\mathrm{Hg}$ y $\mathrm{Pb}$, esto se añade a la evidencia reportada por Tagliari et al. (2004). Como se puede apreciar en la tabla 3, la respuesta de los linfocitos frente a cada una de las dosis del extracto de aguas residuales de curtiembre ocasionó daños, en especial, roturas de cadena sencilla en el ADN. Los resultados hallados en el presente estudio, se pueden explicar teniendo en cuenta que se ha demostrado que la carcinogenicidad del cromo puede ser promovida, a través de varios mecanismos, incluyendo la reducción metabólica intracelular del $\mathrm{CrVI}$, produciendo especies de cromo, capaces de interactuar con el ADN, las cuales, son las responsables de los efectos mutagénicos y genotóxicos (Sedman et al. 2006; Kristen et al. 2010). También, se ha demostrado que el cromo induce aberraciones cromosómicas, rupturas de cadena simple, aductos, sitios AP, oxidación de bases, entrecruzamientos, daño al enlace fosfodiester, daño en los sistemas de reparación, sustitución y delección de bases (Sedman et al. 2006; Salnikow \& Zhitkovich, 2008; O’Brien et al. 2009). Este efecto que produce alteraciones en el material genético es probablemente causado, además, por la presencia de metales, incluyendo el hierro, manganeso, plomo, cobre, zinc y níquel, debido a su alta actividad mutagénica, según fue identificado por Vargas et al. (2001). En la tabla 3, se muestra el daño del ADN inducido por las aguas residuales de la curtiembre Tasajero en Cúcuta, Norte de Santander.

De este estudio, se concluye que los compuestos presentes en las aguas residuales de la curtiembre Tasajero de Cúcuta inducen mutagenicidad en las cepas de Salmonella typhimurium TA98; dicha mutagenicidad, se atribuye a la presencia de compuestos mutagénicos, como metales pesados, cuyo mecanismo molecular, para inducir la mutación, es por perdida o ganancia de bases. Dado que la enfermedad del cáncer es producida por múltiples factores, la exposición a compuestos inherentes a la industria de curtiembres, podría constituir un riesgo en la aparición de dicha enfermedad.

Conflicto de intereses: El manuscrito fue preparado y revisado con la participación de todos los autores, quienes declaramos que no existe conflicto de intereses que ponga en riesgo la validez de los resultados presentados.

\section{BIBLIOGRAFÍA}

1. ACHARYA, S.; MEHTA, K.; KRISHNAN, S.; RAO, C.V. 2001. A subtoxic interactive toxicity study of ethanol and chromium in male Wistar rats. Alcohol. 23:99108.
2. AGENCY FOR TOXIC SUBSTANCES and DISEASE REGISTRY (ATSDR). 2000. Toxicological profile for Polychlorinated Biphenyls (PCBs). p.1-92.

3. ALBERG, A.J.; SAMET, J.M. 2003. Epidemiology of lung cancer. Chest. 123(1 Suppl):21S-49S.

4. ÁlVAREZ-BERNAL, D.; CONTRERAS-RAMOS, S.M.; TRUJILLO-TAPIA, N.; OLALDE-PORTUGAL， V.; FRIAS-HERNANDEZ, J.T.; DENDOOVEN, L. 2006. Effects of tanneries wastewater on chemical and biological soil characteristics. Appl. Soil Ecology. 33:269-277.

5. AMES, B.N.; LEE, F.D.; DURSTON, W.E. 1973. An improved bacterial test system for the detection and classification of mutagens and carcinogens. Proc. Nal. Acad. Sci. (USA). 70(3):782-786.

6. AMES, B.N.; MCCANN, J.; YAMASSAKI, E. 1975. Methods for detecting carcinogens and matagens with the Salmonella/mammalian microsmes muagenicity test. Mut. Res. 31:347-364

7. CASTLEMAN, B. 1995. Bayer, Dow: Migración de riesgos industriales. Rev. del Sur, Octubre 1995. Disponible desde Internet en: http://www.cbgnetwork.org/925. html (con acceso 18/10/14)

8. CERYAK, S.; ZINGARIELLO, C.; O'BRIEN, T.; PATIERNO, S.T. 2004. Induction of pro-apoptotic and cell cycle-inhibiting genes in chromium (VI)-treated human lung fibroblasts: lack of effect of ERK. Mol. Cell. Biochem. 255:139-149.

9. CHÁVEZ PORRAS, A. 2010. Descripción de la nocividad del cromo proveniente de la industria curtiembre y de las posibles formas de removerlo. Rev. Ingenierías. (Colombia). 9(17):41-49.

10. Di IACONI, C.; Di PINTO, A.C.; RICCO, G.; TOMEI, M.C. 2002. Treatment options for tannery wastewater II: integrated chemical and biological oxidation. Ann. Chim. 92(5-6):531-539.

11. DHAYALAN, K.; FATHIMA, N.N.; GNANAMANI, A.; RAO, J.R.; NAIR, B.U.; RAMASAMI, T. 2007. Biodegradability of leathers through anaerobic pathway. Waste Managem. 27(6):760-767.

12. DOTRO, G.; CASTRO, S.; TUJCHNEIDER, O.; PIOVANO, N.; PARIS, M.; FAQQI, A.; PALAZOLO, P.; LARSEN, D.; FITCH, M. 2012. Performance of pilot-scale constructed wetlands for secondary treatment of 
chromium-bearing tannery wastewaters. J. Hazard Mater. 239-240:142-151.

13. De FLORA, S. 2000. Threshold mechanisms and site specificity in chromium (VI) carcinogenesis. Carcinogenesis. 21:533-541.

14. EUROPEAN COMISSION. 2001. European ComissionDirectorate-General for Environment. Survey on Wastes Spread on Land-Final Report. Study contract B4-3040/99/110194/MAR/E3. ISBN 92-894-1732-3. p.1-109.

15. HOLMES, A.L.; WISE, S.S.; WISE, J.P. 2008. Carcinogenicity of hexavalent chromium. Indian J. Med. Res. 128:353-372.

16. JENNETTE, K.W. 1982. Microsomal reduction of the carcinogen chromate produces chromium (V). J. Am. Chem. Soc. 104(3):874-875.

17. KRISTEN, P.; NICKENS, A.; ATIERNO, S.R.; CERYAK, S. 2010. Chromium genotoxicity: A double-edged sword. Chemico-Biological Interactions.188:276-288.

18. LOFRANO, G.; MERIC, S.; ZENGIN, G.E.; ORHON, D. 2013. Chemical and biological treatment technologies for leather tannery chemicals and wastewaters: a review. Sci. Total Environ. 461-462:265-281.

19. MARON, D.M.; AMES, B.N. 1973. Revised methods for the salmonella mutagenicity test. Mutat. Res. 113(34):173-215.

20. NATIONAL TOXICOLOGY PROGRAM. 2005. NTP 11th Report on Carcinogens. Rep. Carcinog. 11:1-A32.

21. O'BRIEN, T.; XU, J.; PATIERNO, S.R. 2001. Effects of glutathione on chromium-induced DNA crosslinking and DNA polymerase arrest. Mol. Cell. Biochem. 222:173-182.

22. O'BRIEN, T.J.; CERYAK, S.; PATIERNO, S.R. 2003. Complexities of chromium carcinogenesis: role of cellular response, repair and recovery mechanisms. Mutat. Res. 533:3-36.

23. O'BRIEN, T.J.; WITCHER, P.; BROOKS, B.; PATIERNO, S.R. 2009. DNA polymerase zeta is essential for hexavalent chromium-induced mutagenesis, Mutat. Res. 663:77-83.
24. PANDRANGI, R.; PETRAS, M.; RALPH, S.; VRZOC, M. 1995. Alkaline single cell gel (comet) ssay and genotoxicity monitoring using bulheads and carp. Environ. Mol. Mutagen. 26(4):345-35.

25. PLUNKETT, E.R. 1976. Handbook of Industrial Toxicology. Chemical Publishing, New York, NY. 536p.

26. SALNIKOW, K.; ZHITKOVICH, A. 2008. Genetic and epigenetic mechanisms in metal carcinogenesis and cocarcinogenesis: nickel, arsenic, and chromium. Chem. Res. Toxicol. 21:28-44.

27. SEDMAN, R.M.; BEAUMONT, J.; McDONALD, T.A.; REYNOLDS, S.; KROWECH, G.; HOWD, R. 2006. Review of the evidence regarding the carcinogenicity of hexavalent chromium in drinking water. J. Environ. Sci. Health. C. Environ. Carcinog. Ecotoxicol. Rev. 24:155-182.

28. SINGH, N.P.; MCCOY, M.T.; TICE, R.R.; SCHNEIDER, E.L.1988. A simple technique for quantitation of low levels of DNA damage in individual cells. Exp. Cell Res. 75(1):184-91.

29. SHAKIR, L.; EJAZ, S.; QURESHI, N.A.; ANJUM, A.A.; IITAF, I.; JAVEED, A. 2012. Ecotoxicological risks associated with tannery effluent wastewater. Environ. I Toxicol. Pharmacol. 34(2):180-191.

30. SUGIYAMA, M.; TSUZUKI, K.; LIN, X.; COSTA, M. 1992. Potentiation of sodium Chromate (VI)-induced chromosomal aberrations and mutation by vitamin B2 in Chinese hamster V79 cells. Mutat. Res. 283:211214.

31. SUGIYAMA, M. 1991. Effects of vitamins on chromium (VI)-induced damage. Environ. Health Perspect. 92:63-70.

32. TAGLIARI, K.C.; CECCHINI, R.; ROCHA, J.A.V.; VARGAS, V.M.F. 2004. Mutagenicity of sediment and biomarkers of oxidative stress in fish from aquatic environments under the influence of tanneries. Mutat. Res. 561:101-117.

33. TÖRNQVIST, R.; JARSJO, J.; KARIMOV, B. 2011. Health risks from large-scale water pollution: trends in Central Asia. Environ. Int. 37(2):435-42.

34. THANIKAIVELAN, P.; RAO, J.R.; NAIR, B.U.; RAMASAMI, T. 2005. Recent trends in leather making: processes, problems, and pathways. Crit. Rev. Environ. Sci. Techn. 35(1):37-79. 
35. U.S. ENVIRONMENTAL PROTECTION AGENCY. 1999. Integrated Risk Information System on Chromium (VI).p.17-29.

36. VARGAS, V.; MIGLIAVACCA, S.; MELO, A.; HORN, R. 2001. Genotoxicity assessment in aquatic environments under the influence of heavy metals and organic contaminants. Mut. Res. 490:141-158.

37. WISE, S.S.; SCHULER, J.H.; HOLMES, A.L.; KATSIFIS, S.P.; KETTERER, M.E.; HARTSOCK, W.J.; ZHENG, T.; WISE, J.P. Sr. 2004. Comparison of two particula- te hexavalent chromium compounds: barium chromate is more genotoxic than lead chromate in human lung cells. Environ. Mol. Mutagen. 44:156-162.

38. WITSCHI, H.R.; PINKERTON, K.E.; VANWINKLE, L.S,; LAST, J.A. 2008. CASSARETT \& DOULL's. Toxicology: The basic science of poisons. chapter 15 . Toxic responses of the respiratory system. $7^{\text {th }}$ edition, ed. McGraw-Hill Inc. New York, NY. p.609-630.

Recibido: Abril 29 de 2014

Aceptado: Diciembre 3 de 2014

\section{Cómo citar:}

Quijano Parra, A.; Castillo T., C.; Meléndez Gélvez, I. 2015. Potencial mutagénico y genotóxico de aguas residuales de la curtiembre tasajero en la ciudad de Cúcuta, Norte de Santander, Colombia. Rev. U.D.C.A Act. \& Div. Cient. 18(1): 13-20. 